\title{
Investigación ecológica participativa como apoyo de procesos de manejo y restauración forestal, agroforestal y silvopastoril en territorios campesinos. Experiencias recientes y retos en la sierra Madre de Chiapas, México
}

\author{
Participatory ecological research supporting forest, agroforest and silvopastoral restoration in \\ peasant territories. Recent experiences and challenges in the Sierra Madre de Chiapas, Mexico \\ Luis García-Barrios $^{\mathrm{a}, *}$ y Mario González-Espinosa ${ }^{\mathrm{b}}$ \\ ${ }^{a}$ Departamento de Agricultura, Sociedad y Ambiente, El Colegio de la Frontera Sur, Carretera Panamericana y Periférico Sur s/n, Barrio de María Auxiliadora, \\ 29290 San Cristóbal de Las Casas, Chiapas, México \\ b Departamento de Conservación de la Biodiversidad, El Colegio de la Frontera Sur, Carretera Panamericana y Periférico Sur s/n, Barrio de María Auxiliadora, \\ 29290 San Cristóbal de Las Casas, Chiapas, México
}

Recibido el 2 de marzo de 2016; aceptado el 4 de octubre de 2016

Disponible en Internet el 14 de noviembre de 2017

\begin{abstract}
Resumen
Quienes realizamos investigación ecológica forestal y agroforestal reconocemos crecientemente la indisoluble interacción entre los procesos ecológicos y sociales, y nos vamos sumando a los esfuerzos de investigación multi- y transdisciplinaria y de acción entre múltiples actores sociales. Esto ha modificado en diversos sentidos y grados nuestros objetivos, conceptos, escalas, métodos y prácticas. La investigación participativa ecológica forestal y agroforestal (IPEFA) se concibe y se practica de muy distintas maneras, definidas por voluntad de quienes investigan y/o por las circunstancias que enfrentan. Aquí presentamos y reflexionamos nuestra experiencia directa en 2 procesos de IPEFA que hemos coordinado en la última década en territorios campesinos pobres y medios de la Sierra Madre de Chiapas: la cuenca alta del río El Tablón en la Reserva de la Biosfera de La Sepultura (LGB) y las cuencas de los ríos Xelajú y Bacantón (alto Grijalva), en los municipios de Motozintla y Mazapa (MGE). Presentamos logros, pero sobre todo destacamos algunos de los mayores retos que enfrentamos y aquellos caminos que no siempre llegaron a buen puerto. Creemos que varios de estos retos académicos, sociales, e institucionales son compartidos por la comunidad mexicana e internacional que practica la IPEFA y que se esfuerza continuamente por superarlos.
\end{abstract}

(C) 2017 Universidad Nacional Autónoma de México, Instituto de Biología. Este es un artículo Open Access bajo la licencia CC BY-NC-ND (http://creativecommons.org/licenses/by-nc-nd/4.0/).

Palabras clave: Investigación participativa; Restauración forestal; Agroforestería; Sierra Madre de Chiapas; Ecología en México

\begin{abstract}
Those of us who conduct forest and agroforest ecological research increasingly recognize the interaction between ecological and social processes and join multi and transdisciplinary research and action efforts involving multiple stakeholders. This has changed in various degrees our goals, concepts, scales, methods and practices. Ecological participatory forestry and agroforestry research (IPEFA) is conceived and practiced in many different ways, defined by the will of those who investigate and (or) the circumstances they face. Here we present and make a reflection of our direct experience in two IPEFA processes we have coordinated in the last decade in poor rural territories of the Sierra Madre de Chiapas: the upper basin of El Tablón River in the Biosphere Reserve La Sepultura (LGB), and the basins of the rivers Xelajú and Bacantón (High Grijalva), in the
\end{abstract}

\footnotetext{
* Autor para correspondencia.

Correo electrónico: luis.garciabarrios@ gmail.com (L. García-Barrios).
}

La revisión por pares es responsabilidad de la Universidad Nacional Autónoma de México. 
municipalities of Motozintla and Mazapa (MGE). We present achievements, but above all highlight some of the biggest challenges we have faced, and those roads that did not always lead to good results. We believe that several of these academic, social, and institutional challenges are shared by Mexican and international researchers that practices IPEFA and continually strive to overcome them.

(C) 2017 Universidad Nacional Autónoma de México, Instituto de Biología. This is an open access article under the CC BY-NC-ND license (http://creativecommons.org/licenses/by-nc-nd/4.0/).

Keywords: Participatory research; Forest restoration; Agroforestry; Sierra Madre de Chiapas; Ecology in Mexico

\section{Introducción}

En las extensas cordilleras neotropicales de México se mantienen territorios campesinos con distintos grados de cobertura arbolada en un intrincado mosaico con fragmentos del paisaje bajo diferentes usos de tipo forestal, silvopastoril, agroforestal y agrícola. A pesar de profundas transformaciones recientes, los modos predominantes de vida en estas regiones se caracterizan por su tendencia al minifundio, por su escasez de empleo y de recursos para invertir en la producción, así como por la persistencia de condiciones desfavorables de intercambio en los mercados regionales y globales. Estos modos de vida han dependido y mantendrán su dependencia del conocimiento y manejo campesino de los procesos ecológicos que ocurren en los ecosistemas forestales, agroforestales y silvopastoriles (Astier, García-Barrios, Masera-Cerutti, Galván-Miyoshi y GonzálezEsquivel, 2012; Chappell et al., 2013; Perfecto y Vandermeer, 2015). Las consecuencias favorables y desfavorables de este patrón de aprovechamiento de los recursos se extienden a otros grupos sociales tales como la población rural que vive en las partes bajas de las cuencas, la población de centros urbanos aledaños y, en general, la población afectada por cambios climáticos regionales y globales.

Quienes practicamos la ecología e investigamos los ecosistemas de montaña hemos transitado en unas pocas décadas de practicar predominantemente una ciencia básica orientada a documentar y entender la composición, estructura, dinámica y productividad de los mismos, a convocar o atender la convocatoria de colegas de otras disciplinas y actores sociales, interesados en aportar - a escala local, nacional o global- a la gestión o transformación de las crisis socioambientales contemporáneas en general, y a sus manifestaciones particulares en los territorios que aquí nos atañen. Reconocer la indisoluble interacción entre los procesos ecológicos y sociales y sumarnos a esfuerzos de investigación multi- y transdisciplinaria y de acción entre múltiples actores sociales ha modificado en diversos sentidos y grados nuestro marco de referencia para la investigación ecológica, y en consecuencia, nuestros objetivos, conceptos, escalas, métodos y prácticas. En el camino hemos acuñado, adoptado o debatido críticamente paradigmas socioecológicos como sustentabilidad, conocimiento ecológico tradicional, justicia ambiental, complejidad/resiliencia/adaptabilidad socioecológica, optimización de servicios ecosistémicos, pago por servicios ambientales, desarrollo de industria/mercados/capitales verdes, etcétera, cuyo origen ideológico y político, así como sus consecuencias prácticas en lo ecológico, económico, social y cultural no siempre alcanzamos a vislumbrar plenamente. En el amplio abanico de posiciones que genera el debate de objetivos y paradigmas de investigación y acción, pareciera haber un punto de consenso: la idea de que es conveniente, o incluso indispensable, la participación de la mayoría de los actores involucrados en la investigación/acción orientada a la llamada gobernanza de procesos socioecológicos (Fortmann, 2008). Sin embargo, los objetivos, los actores, los métodos, así como las relaciones de poder que conducen esta participación son también muy diversos y sujetos a intenso debate (Newig y Fritsch, 2009).

En el caso de lo que podemos llamar la investigación participativa ecológica forestal y agroforestal (IPEFA) en territorios campesinos montañosos neotropicales, la posible participación de la población local, y de los actores que interactúan de manera directa e inmediata con ella, se concibe y se practica de muy distintas maneras, con perspectivas y niveles de compromiso muy variados. Podemos mencionar, de manera no exhaustiva: desde la extracción de información a la población local, la consulta apresurada al inicio y el final de las investigaciones, el monitoreo participativo de contratos comerciales de captura de carbono, hasta el acompañamiento científico multidisciplinario de procesos de largo plazo dirigidos realmente por los campesinos empoderados, actores directos y decisores sobre el uso de sus recursos y territorios, así como de los posibles cambios en sus modos de vida comunitarios y su cultura

En el campo de la IPEFA se han postulado historias de éxito y casos sobresalientes en el mundo y en México (p. ej. Velázquez, Torres y Bocco, 2003); no obstante, predomina la creciente dificultad para llevar a buen puerto estas iniciativas y sostenerlas en el largo plazo. Esto es particularmente cierto donde y cuando se llegan a generar tensiones en los territorios rurales debido al extremo deterioro social y ambiental y/o donde hay fuertes disputas (abiertas o veladas) por el territorio y sus ecosistemas (p. ej. las diferentes versiones de las áreas naturales protegidas). Las dificultades pueden comprenderse mejor si se analiza cómo se entrelazan las dinámicas que imponen los modelos de economía y sociedad imperantes, las agendas de investigación y desarrollo que proponen implícita o explícitamente las instituciones de gobierno y la sociedad civil hacia los procesos de aprovechamiento forestal y agroforestal, así como la manera en que los investigadores conciben e implementan la IPEFA.

Es necesario analizar en qué medida la agenda predominante de las políticas forestales y de la IPEFA en México, así como las modalidades de participación que promueve, contri- 
buyen a aumentar o reducir estas dificultades, a menudo no previstas en los paradigmas y modelos adoptados, que se definen en otros países, como lo hacen Larson y Ribot (2007) para las políticas forestales en Honduras. En este artículo nos limitaremos a presentar y reflexionar la experiencia directa en 2 procesos de IPEFA que hemos tenido la oportunidad de coordinar en la última década en territorios campesinos pobres y medios de la Sierra Madre de Chiapas: la cuenca alta del río El Tablón (CART) en la Reserva de la Biosfera de La Sepultura (LGB), y las cuencas de los ríos Xelajú y Bacantón, en la parte fronteriza con Guatemala de la cuenca del río Grijalva, en los municipios de Motozintla de Mendoza y Mazapa de Madero (MGE).

Expondremos brevemente lo hecho, su impacto actual y potencial, y reflexionaremos sobre lo aprendido, con la esperanza de que quien lea este trabajo encuentre preguntas y respuestas útiles para rectificar lo que no funciona, así como fortalecer lo que sí. Trataremos de seguir un guión relativamente común para presentar los hechos que constituyen cada caso; en la parte final de balance del documento nos enfocaremos en los retos comunes que enfrenta la IPEFA en la Sierra Madre de Chiapas y posiblemente en otros territorios campesinos social y ecológicamente similares.

En ambos casos, partimos de reconocer que nuestro mayor reto en la práctica o implementación de la investigación ecológica participativa no es tanto sobre cómo hacer participar a los actores rurales de un territorio en nuestras investigaciones ecológicas, sino cómo aprender a participar como ecólogos en los procesos fundamentales requeridos para la gestión territorial de parte de esos actores, en primera instancia en beneficio de la población local.

\section{Investigación ecológica participativa en la cuenca alta del río El Tablón (2006-2015)}

\section{Un panorama sucinto de algunos procesos que definen la interacción entre actores que disputan la gestión de este territorio originalmente forestal}

\section{La cuenca alta del río El Tablón, un territorio muy dinámico y en disputa}

A 10 años de haber iniciado nuestras investigaciones, se ha alcanzado cierta claridad sobre procesos territoriales relevantes, algunos de los cuales se sintetizan aquí (Cruz-Morales, 2014a, b; Cruz-Morales, Trujillo-Vázquez, García-Barrios, Ruiz-Rodríguez y Jiménez-Trujillo, 2011), y se basan en los estudios reseñados en García-Barrios et al. (2012) y en la sección «Una vista general de nuestro proceso de inserción como investigadores en esta dinámica» de este artículo. La CART se ubica en el sotavento de la porción noroeste de la Sierra Madre de Chiapas y forma parte de la cuenca suroeste del río Grijalva. Es un espacio de aproximadamente 30,000 ha, montañoso y muy abrupto, con un gradiente climático significativo (altitudes de 800-2,550 m; con clima templado húmedo hasta semicálido subhúmedo); incluye una densa red hídrica permanente e intermitente que crea valles aluviales muy estrechos; suelos forestales transformados, de origen granítico;
6 tipos de bosques, predominantemente selva baja caducifolia, bosque de pino-encino y bosque mesófilo de montaña, y en conjunto, una altísima biodiversidad. Hasta 1960, este espacio montañoso fue de «tierras nacionales», usadas marginal, ilegal e intermitentemente por empresas forestales medianas y por ranchos ganaderos aledaños. La población construyó un territorio campesino, agrícola y ejidal cuyo motor económico y político fundamental fue, hasta fines de los ochenta, la incorporación al «boom» de producción moderna y comercial de maíz de La Fraylesca. La población mantuvo como actividades mercantiles secundarias la ganadería trashumante en los bosques, y la extracción de palma Chamaedorea spp. y de madera. A mediados de los noventa, una tenaza múltiple aprisionó los modestos medios y estrategias de vida de la mayoría de la población en la CART: a) se reformó el artículo 27 constitucional y se dio fin al reparto agrario y a la ampliación ejidal; $b$ ) se decretó toda la CART como parte de la Reserva de la Biosfera La Sepultura (REBISE) y se prohibieron el desmonte, la extracción forestal y el uso silvoagropecuario del fuego; y $c$ ) se desplomó la rentabilidad del cultivo de maíz con la importación masiva de este grano. La población de los 12 ejidos de la CART reorganizó sus estrategias: encontró en la compra de ganado, la expansión de potreros y el pastoreo más intenso en los bosques la forma de adaptación por excelencia a las nuevas circunstancias. Los incendios sin precedentes del año 1998 asociados al severo fenómeno ENSO (Román-Cuesta, Gracia y Retana, 2003) y a la expansión del hato bovino en la REBISE, motivaron a la Conanp, a la Comisión Nacional Forestal (Conafor) y a las corporaciones internacionales de conservación a condenar la ganadería extensiva y a promover el manejo integrado del fuego, el pago por servicios hidrológicos y, en las zonas más altas, el cultivo de café de sombra y palma Chamaedorea para mercados internacionales. Al mismo tiempo, se incrementaron los programas de asistencia monetaria al consumo no productivo de alimentos y bienes industrializados. A casi 60 años del inicio de la colonización ejidal, la mayoría de los cerca de 7,000 habitantes de la CART son productores semiespecializados, siempre listos a diversificar, con una moda de 20 ha de tierra en la zona más ganadera y de 5 ha en la más cafetalera; están socialmente polarizados por su actividad preponderante y por su cantidad y tipo de tenencia de la tierra. Los ejidos están formalmente representados en el Consejo Asesor de la REBISE, pero esta instancia tiene poca capacidad de convocatoria y pocas iniciativas propias. El presupuesto y personal de la Conanp en la REBISE es insuficiente, ha estado estancado y recientemente experimentó una reducción significativa. Hoy la CART es un territorio con un mosaico muy intrincado de usos del suelo y tipos de cobertura vegetal, en el que coexisten bosques mesófilos remotos poco perturbados, bosques semiabiertos y pastoreados, acahuales en estados sucesionales tempranos, cafetales de sombra, maizales, y potreros dominados por el zacate introducido jaragua (Hyparrenhia rufa), los cuales comúnmente son sobrepastoreados y mantienen pocos árboles; incluso los suelos de algunos potreros están muy erosionados. La resinación de pinos comienza para empresas privadas en 3 ejidos, en tanto que el café está devastado por la roya y los recolectores de palma buscan nuevos mercados tras cambios en la demanda de su producto. La ganadería basada 
en la cría de becerros para la exportación está nuevamente en expansión.

Una vista general de nuestro proceso de inserción como investigadores en esta dinámica

\section{Evolución de nuestras formas de participar como investigadores en el proceso de la cuenca alta del río El Tablón}

En 2005, a partir de experiencias internacionales de investigación como «Aridnet» en Honduras, con respecto al desarrollo social e innovación agroecológica, que tenía como eje la promoción del policultivo de maíz, frijol, sorgo y árboles, conocido como «Quesungual» (Ayarza et al., 2010) y del consorcio internacional «Diversitas» y su red de investigación de agrodiversidad (Jackson et al., 2012), se buscó un territorio social y ambientalmente similar en Chiapas en el que se pudiera reproducir la experiencia hondureña, a una escala mucho más modesta, y canalizar así las ideas en boga de la agroecología, la biodiversidad funcional, los paisajes agrodiversos para la conservación y del desarrollo social campesino. Tras meses de buscar intensamente llegamos a la CART. Convocamos a asamblea en el ejido más grande y nuestro invitado —el líder histórico del proyecto «Quesungual» - expuso ante una centena de productores las bondades y logros de 15 años del proyecto.

En la CART hubo que reorientar el proyecto hacia lo silvopastoril iniciando un intenso trabajo de análisis participativo y experimentación campesina en torno al cultivo de árboles forrajeros locales en potreros, encaminado a contribuir a solucionar el problema de escasez de forrajes durante el estiaje. Este proceso trajo a su vez nuevas sorpresas que pusieron de manifiesto razones sociales y ecológicas para cuestionar la validez y viabilidad del cultivo de árboles forrajeros en este territorio.

Esta investigación participativa durante el período 2007-2016 ha contribuido a:

a) explicar los procesos y fuerzas motrices que han conducido a cambios frecuentes en la apropiación y uso del suelo (Speelman et al., 2014; Valdivieso-Pérez, García-Barrios y Plascencia, 2009; Zabala, 2015), y sus consecuencias sobre la composición florística (Valencia, García-Barrios, West, Sterling y Naeem, 2014; Valencia, Naeem, García-Barrios, West y Sterling, 2016; Valencia, West, Sterling, García-Barrios y Naeem, 2015), la cubierta vegetal (Sanfiorenzo-Barnhard, 2012; Sanfiorenzo-Barnhard, García-Barrios, Meléndez-Ackerman y Trujillo-Vásquez, 2009) y la calidad del suelo (Valdivieso-Pérez, García-Barrios, Álvarez-Solís y Nahed-Toral, 2013); b) revelar el bajo nivel de consenso actual entre los actores locales y externos, y al interior de ambos grupos, respecto de cuáles son los recursos más importantes del territorio y sus problemas prioritarios (Brunel-Manse y García-Barrios, 2011); c) comprender a fondo las formas en que la gente practica la ganadería, los problemas que enfrenta, y el lugar que le dan a las prácticas silvopastoriles en su búsqueda de soluciones (García-Barrios et al., 2012; Gutiérrez-Navarro, 2015; Rosabal-Ayan, 2015; Zabala, Pascual y García-Barrios, 2017); d) construir consensos y capacidades sobre cuáles son las mejores prácticas ganaderas y de uso del suelo en el área, con base en el conocimiento local y acadé- mico (Cruz-Morales et al., 2011); e) motivar a 200 campesinos ganaderos a establecer pequeños módulos experimentales con árboles forrajeros y otras especies en sus predios (Cruz-Morales, 2014a, b; García-Barrios et al., 2012); f) analizar las formas en las que los productores participan en estos experimentos y revelar por esta vía las limitaciones ecológicas y los obstáculos y oportunidades sociales para innovar las prácticas ganaderas en la coyuntura actual; $g$ ) desarrollar experimentalmente prácticas agroecológicas que pudieran reducir las restricciones técnicas y económicas que identifican los ganaderos para que la innovación silvopastoril sea atractiva y exitosa (Buhmann y García-Barrios, 2014; Braasch, García-Barrios, RamírezMarcial, Huber-Sannwald y Cortina-Villar, 2017; Morales-Díaz, 2011; Oleta-Barrios, 2012; Vides-Borrell et al., 2011); $h$ ) involucrar a los actores locales y externos en construir juntos un proceso de educación popular a mediano plazo. El énfasis ha estado en diseñar y usar juegos ecológicos y socioecológicos (de mesa y computadora) que aporten elementos a la población local para innovar y adaptar sus normas comunitarias, sus prácticas agrícolas y sus modos de vida semiespecializados en ganadería, resina o café a los nuevos retos y oportunidades ecológicos y sociales que enfrenta (García-Barrios, García-Barrios, Cruz-Morales y Smith, 2015; García-Barrios, García-Barrios, Waterman y Cruz-Morales, 2011; Meza-Jiménez y GarcíaBarrios, 2015; Meza-Jiménez, García-Barrios, Saldívar-Moreno y Vera-Noriega, 2016).

\section{Un ejemplo de la inevitable dinámica de encuentros y desencuentros en la investigación participativa ecológica forestal y agroforestal}

\section{Cultivo de forrajes y conservación del bosque. Dinámica de un proceso de investigación acción participativa multiactor}

Por definición (Salazar, 1992), la investigación acción participativa multiactor en el medio rural no es un proceso terso; es un esfuerzo constante por hacer más transparente y democrático el juego de los intereses, tiempos, procedimientos y estilos de actores que son social y culturalmente diferentes. La investigación acción participativa multiactor para explorar la viabilidad ecológica y social del cultivo de árboles forrajeros en potreros de la CART lo atestigua (García-Barrios, Trujillo-Vásquez, CruzMorales, Waterman y García-Barrios, 2010; García-Barrios et al., 2012). Sucintamente, esta ha pasado hasta ahora por 4 etapas: a) en 2007, ECOSUR motivó a un grupo de pequeños ganaderos de uno de los ejidos para que establecieran de manera voluntaria, y sin recibir incentivos monetarios, 20 pequeñas parcelas para explorar el desempeño de especies arbóreas forrajeras locales asociadas a los potreros. La Conanp se interesó en el proceso y formó un grupo paralelo en el mismo poblado. Ambas instituciones invitaron a los productores a coordinarse para la producción del almácigo, pero se suscitaron tensiones por diferencias previas entre los grupos. ECOSUR monitoreó en gran detalle las 40 parcelas y reportó abiertamente a los productores que una proporción importante de ellos dejó morir los árboles sin sembrarlos, que la mayoría tuvo árboles agobiados por el pasto, y que una pequeña minoría tuvo resultados excelentes; $b$ ) entre 2008 y 2010, ECOSUR convocó nuevamente a los 40 ganaderos 
a establecer de manera voluntaria pequeñas parcelas experimentales de la especie más promisoria, en tanto que Conanp ofreció recursos a fondo perdido, monetarios y en especie. ECOSUR monitoreó las parcelas y encontró el mismo nivel de abandono y de bajo desempeño de los árboles, mismo que reportó al grupo. Inició entonces estudios participativos para identificar las causas sociales, técnicas y ecológicas de estos resultados; $c$ ) en 2011, el grupo de ECOSUR y la UACH retomaron la iniciativa, discutieron con los productores, y se acordó establecer nuevos experimentos manejados por ellos con un formato de concurso amistoso con premios a los 10 productores que tuvieran las mejores plántulas forrajeras al cabo de un año. No se otorgaron recursos incondicionales y los premios no consistieron en dinero para el consumo no productivo; se otorgaron reconocimientos públicos, ejemplares de un libro sobre buenas prácticas ganaderas elaborado con su participación (Cruz-Morales et al., 2011), y equipo y una pequeña picadora de forrajes para los primeros lugares. El evento de premiación fue la ocasión para nuevamente presentar y discutir los resultados del monitoreo ecológico, al que asistieron representantes de los 12 ejidos de la CART, la Conanp y varias instituciones y organismos no gubernamentales. Con este antecedente, se convocó a grupos interesados de los 12 ejidos y a la Conanp, la organización AMBIO, la UACH y ECOSUR a coordinarse para generar en 2012 una experiencia de construcción de módulos silvopastoriles que incluyeran las propuestas de todas las instituciones que hasta el momento competían por la atención de los ganaderos (árboles forrajeros, pastos de corte, árboles maderables y la leguminosa herbácea forrajera Canavalia ensiforme). En talleres, los productores diseñaron las parcelas a construir en áreas de ladera y entre todos los participantes se establecieron las reglas del juego para un nuevo concurso amistoso a escala de la CART, con reconocimientos y premios numerosos de carácter productivo. De los resultados obtenidos por los 120 productores participantes, destaca que ya no hubo abandono de las parcelas, el $20 \%$ tuvieron módulos evaluados por el colectivo como insuficientes; el $60 \%$ como aceptables y el $20 \%$ como excelentes. En los módulos, la especie que generó mayor interés y atenciones fue el pasto cubano bajo riego (Pennisetum purpureum $\times$ Pennisetum thyphoides) porque produjo abundante biomasa en corto tiempo; le siguieron los bancos de proteína de árboles forrajeros y $C$. ensiforme; $d$ ) entre 2013 y 2015, la Conanp se separó de esta red y traspasó los esfuerzos y logros de la misma a una colaboración con el Centro Agronómico Tropical de Investigación y Enseñanza (CATIE) y The Nature Conservancy (TNC) para continuar la promoción del proyecto silvopastoril en el marco de un programa para la REBISE, con fuerte financiamiento internacional relacionado con cambio climático. En los hechos, el trabajo de este programa en la CART consistió en otorgar insumos de riego para cultivar pasto cubano en vegas de ríos a una pequeña porción de los participantes que tenía producción lechera semiestabulada, lo que aceptaron a cambio de «liberar a la conservación» sus predios de bosques semiabiertos en los que hasta ahora ramoneaba estacionalmente su ganado. Esta estrategia de la Conanp (cultivo intensivo de pastos de corte es más importante que el establecimiento de arboledas en los potreros) es compatible con su plan de manejo y zonificación de la REBISE, que busca en última instancia -en apego al modelo de configuración territorial divergente (explicado en García-Barrios et al., 2009; TenzaPeral, García-Barrios y Giménez-Casalduero, 2011) - separar en lo posible la producción y la conservación dentro de la REBISE. Este esquema de manejo es crecientemente señalado como inapropiado para muchos de estos territorios reservados (Sayer et al., 2013). Los grupos de la UACH y ECOSUR concentraron sus recursos en seguir la exploración de otras posibilidades del cultivo de árboles forrajeros en las laderas en las que tienen sus tierras la mayoría de los ganaderos (p. ej. Buhmann y García-Barrios, 2014) y en atender otros aspectos de la IPEFA en la CART.

\section{Ejemplos de lo que podemos aportar desde la disciplina de investigación ecológica}

\section{El reclutamiento de árboles cultivados y silvestres en la cuenca alta del río El Tablón: desafío y tema recurrente de investigación ecológica participativa}

Los usos del suelo que actualmente se perfilan como capaces de crear paisajes silvopastoriles y agroforestales en la CART, compatibles con los intereses de la población y de la Conanp, tienen en común un problema de reclutamiento y/o permanencia a largo plazo de las especies arbóreas que los componen. Es el caso de: a) la creciente dificultad ecológica para establecer plantaciones de árboles forrajeros locales entre especies de pasto muy competitivas en un ambiente que, al parecer, se torna cada vez más árido (García-Barrios et al., 2012); b) el casi nulo reclutamiento de pino resinero (Pinus oocarpa) en presencia de pastos competitivos, ausencia de fuego y efectos múltiples del pastoreo excesivo en los rodales bajo resinación; c) la substitución paulatina de la diversidad de árboles silvestres del bosque mesófilo en las plantaciones de café por el árbol chalum (Inga spp.) (Valencia et al., 2014, 2015, 2016) y la falta de reclutamiento en estas plantaciones y en las de palma bajo sombra.

Se trata de procesos ecológicos sutiles y con consecuencias a largo plazo, a los que tanto la Conanp como los productores de la CART les prestan hoy poca atención por atender las urgencias y prioridades del presente. Se describen brevemente las investigaciones ecológicas sobre los puntos $a$ ) y $b$ ), propiamente de corte silvopastoril.

\section{Establecimiento de árboles forrajeros}

Los productores seleccionaron 4 especies arbóreas locales propias del bosque tropical seco o selva baja caducifolia para evaluar experimentalmente su establecimiento, crecimiento juvenil y palatabilidad. De ellas, la que se desempeñó mejor fue Gliricidia sepium (mataratón, madrecacao, cocoíte). Los experimentos de algunos participantes han demostrado que las plantaciones de esta especie se pueden establecer exitosamente. Muchos productores encuentran demasiado laborioso y costoso producir y acarrear plantas de almácigo y realizar limpias. Una serie de experimentos ecológicos en predios de los productores han mostrado que el crecimiento en siembra por semilla es viable aunque significativamente más lento en términos absolutos y relativos que por trasplante desde almácigo, lo cual las hace más vulnerables a la competencia y demanda limpias adicio- 
nales (Morales-Díaz, 2011); si se deshierba apropiadamente la plantación por un tiempo, el dosel cerrado de G. sepium es capaz de excluir por completo a los pastos más competitivos y dejar crecer herbáceas umbrófilas de porte alto, potencialmente forrajeras (Buhmann y García-Barrios, 2014; Morales-Díaz, 2011); en ausencia de suficiente deshierbe, el pasto agobia al árbol joven y lo hace en mayor medida si se aplican fertilizantes sintéticos en vez de orgánicos (Vides-Borrell et al., 2011).

\section{Reclutamiento de pino resinero}

Algunos ejidos de la CART tienen bosques de $P$. oocarpa semiabiertos, sometidos a pastoreo. Hasta hace pocos años se suscitaron conflictos serios entre los productores y la Conanp debido a la necesidad de quemar los potreros y el deseo de aprovechar la madera. Tras varios años de gestiones, los productores lograron un permiso de resinación que ha conciliado por ahora los intereses de varios actores; los resineros han suspendido por interés propio el uso del fuego y algunos han excluido el ganado de sus predios. ECOSUR se acercó a estos productores y a otros actores involucrados para conocer sus intereses e inquietudes. De ello surgió un interés compartido en explorar si las condiciones ecológicas que crea esta nueva actividad y su manejo pueden afectar el reclutamiento de las plántulas de $P$. oocarpa que son necesarias para sustituir a los adultos cuando estos últimos agoten su vida productiva de aproximadamente 20 años. Se han iniciado desde 2015 varias actividades de investigación participativa (Braasch et al., 2017) que incluyen: $a$ ) inventarios forestales realizados con y por los productores; $b$ ) experimentos en rodales de los productores para dilucidar los efectos sobre el reclutamiento que resultan de la interacción entre los pastos, el régimen de fuego y la intensidad de pastoreo/pisoteo. Se anticipa que un pastoreo apropiado puede liberar a la plántula de la competencia intensa de pastos no controlados por el fuego; y c) modelos amigables de simulación de agentes ecológicos y sociales, parametrizados con los resultados del estudio, para que los actores interesados exploren conjuntamente las consecuencias de diversas estrategias de manejo silvopastoril de los rodales resineros.

\section{Ejemplos de lo que podemos aportar desde la interdisciplina de investigación socioecológica}

\section{Construcción de aprendizajes socioecológicos entre los actores mediante juegos de mesa y simulaciones}

Se inició la tarea de extender nuestra experiencia previa en modelación ecológica para construir con los actores de la CART juegos de mesa socioecológicos que capturaran problemas definidos por ellos en talleres realizados para este fin. Estos juegos capturan procesos identificados por varios actores y los estructuran y estilizan con elementos de la teoría de los dilemas de lo colectivo (Ostrom, 2009); la teoría economicopolítica de juegos estratégicos cooperativos y no cooperativos (Tadelis, 2013); la teoría del institucionalismo crítico (Van Hecken, Bastiaensen y Windey, 2015); los principios y métodos de la modelación acompañante (Etienne, 2014), y la teoría de procesos complejos (Gershenson, Aerts y Edmonds, 2007).
Entre 2007 y 2010 se generaron 3 juegos de gestión social y ecológica de territorios: «Manantiales de la sierra» (GarcíaBarrios et al., 2011, 2015), «Lasarus» (García-Barrios, 2010) y «Resortes» (Speelman, García-Barrios, Groot y Tittonell, 2013). El primero ha sido jugado en talleres colectivos en todos los ejidos de la CART, en el Consejo Asesor de la REBISE, entre personal de la Conanp y entre este y los productores, así como por cientos de estudiantes de posgrado y académicos.

Entre 2013 y 2016 se generó, validó y puso a disposición de las familias cafetaleras de la CART y otras regiones de la Sierra el juego estratégico Ajedrez Azteca (García-Barrios, Perfecto y Vandermeer, 2016; García-Barrios, Cruz-Morales, Vandermeer y Perfecto, 2017) para el aprendizaje social de procesos ecológicos de regulación autónoma de la roya del café (Perfecto y Vandermeer, 2015). Los resultados y aprendizajes generados pueden ser consultados en la literatura citada.

En 2012 se decidió mirar hacia la niñez y adolescentes de la CART quienes muy pronto heredarán y serán responsables del uso y manejo del patrimonio agrícola/natural (PAN) de sus familias y ejidos (Meza-Jiménez, 2012). Coincidimos con quienes buscan desarrollar una práctica crítica y alternativa de la educación ambiental en México (p. ej. Castillo y GonzálezGaudiano, 2009). Por ello, se decidió no inducir conductas sobre lo que un poblador rural «debe» hacer con sus tierras y los seres que en ellas viven, sino generar métodos y herramientas que les permitieran analizar sus propias motivaciones, valores y opiniones. Para ello desarrollamos experiencias teatrales (Heras, Tabara y Meza, 2016) y juegos que les permitieran descubrir y discutir entre ellos la manera en que perciben y desearían manejar este patrimonio, en el contexto más amplio de los desafíos ecotécnicos y dilemas de cooperación que pronto enfrentarán para construir estrategias de vida en la condiciones actuales de crisis rural. De ello surgieron «Los Juegos del PAN (Patrimonio Agrícola/Natural)» (Meza-Jiménez y García-Barrios, 2015; Meza-Jiménez et al., 2016) que incluyen «Los discursos juveniles del PAN», «Mi Territorio», «CargaPalito» y una adaptación de «Manantiales de la sierra (García-Barrios et al., 2011)». Estos han sido aprendidos por un primer grupo de docentes de las telesecundarias rurales de la CART y zonas aledañas, y utilizados con sus estudiantes. Esta acción es muy reciente y quedan por evaluar más adelante si su uso se ha extendido y el posible impacto educativo.

\section{Investigación ecológica y social participativa para la restauración forestal en la cuenca alta del río Grijalva en la Sierra Madre de Chiapas, México}

\section{Descripción general del área de estudio en la cuenca alta del Grijalva}

La cuenca alta del río Grijalva abarca porciones de México y Guatemala (Laíno-Guanes et al., 2016). De acuerdo con el «Simulador de flujos de agua de cuencas hidrográficas» del Instituto Nacional de Estadística y Geografía (SIATL) (Inegi, 2013), la altitud en la región varía desde 420 hasta $2,850 \mathrm{~m}$, con pendientes de $11-12^{\circ}$. Los registros de estaciones meteorológicas obtenidos desde 1978 en Motozintla de Mendoza 
y Buenos Aires indican que el promedio de lluvia anual es de 820 a 1,280 mm, con una estación lluviosa que abarca de mayo a octubre, con $900 \mathrm{~mm}$ en la parte baja pero de hasta más de 3,000 mm en algunas partes altas (Inegi, 1985; Conagua, 2013). La temperatura promedio anual puede ser de $14{ }^{\circ} \mathrm{C}$ en las partes altas y de hasta $24^{\circ} \mathrm{C}$ en la partes más bajas. Los suelos incluyen acrisoles, cambisoles, regosoles y andosoles (Inegi, 1988). Las cuencas de los ríos Xelajú (totalmente incluida en territorio mexicano) y Bacantón (la cuenca es transfronteriza entre México y Guatemala) incluyen unos $120 \mathrm{~km}^{2}$. La porción mexicana de estas 2 cuencas fue estudiada por un equipo multidisciplinario encabezado por ECOSUR entre 2010 y 2013 (González-Espinosa y Brunel-Manse, 2014). Mayores detalles sobre el área de estudio aparecen en Gómez-Pineda et al. (2014), Laíno-Guanes et al. (2015) y Laíno-Guanes, Musálem-Castillejos, González-Espinosa y Ramírez-Marcial (2014).

A principios del siglo pasado los recursos naturales más importantes en la porción fronteriza de la Sierra Madre de Chiapas incluían varios tipos de ecosistemas forestales (Waibel, 1946). Estos bosques incluían selvas bajas caducifolias en las partes bajas y bosques mixtos de pino-encino, bosques maduros de encino y bosques de niebla (o mesófilos de montaña) en las laderas altas y cimas (Breedlove, 1981; González-Espinosa y Ramírez-Marcial, 2013), intensamente talados para aprovechar su madera. La deforestación continuó muy alta hasta tiempos recientes. Villafuerte-Solís (2010) señala que la cobertura forestal disminuyó de 183,000 en 1990 a 53,000 hectáreas en 2005. Junto con la degradación ambiental en la región, las pendientes muy abruptas han incrementado la vulnerabilidad social y económica de los asentamientos dispersos en las partes altas de la región, así como mayores riesgos de deslizamientos de laderas y cambios de cursos de los ríos e inundaciones en las partes bajas de las cuencas, como en el caso de la mayor concentración de población de la región, la ciudad de Motozintla de Mendoza (Caballero et al., 2006; Gómez-Pineda et al., 2014; HernándezMoreno, 2011; Richter, 2000; Villafuerte-Solís y Mansilla, 2010).

Aunque la región fue incorporada a México en 1882 (Villafuerte-Solís y García-Aguilar, 2004), los municipios de Motozintla de Mendoza y Mazapa de Madero fueron más intensamente colonizados a partir del inicio del siglo pasado. Su crecimiento y desarrollo se debió a su función como centro de contratación de empleados temporales de las etnias originarias mam y mochó, así como otras que ahí acudían desde regiones lejanas de Chiapas y Guatemala, requeridos por las fincas cafetaleras de dueños alemanes de Guatemala y el Soconusco (Richter, 2000; Roblero-Morales y Ramírez-Moreno, 2014; Villafuerte-Solís y García-Aguilar, 2004; VillafuerteSolís y Mansilla, 2010). Aunque en la región no se presentaron movimientos campesinos que pudieran modificar la estructura agraria como resultado del final de la Revolución Mexicana (Roblero-Morales y Ramírez-Moreno, 2014), los trabajadores temporales se establecieron en áreas marginales para asegurar la mano de obra requerida por el proceso productivo cafetalero.

\section{Inicio del trabajo en la región}

En 2010 se inició una investigación participativa bajo el enfoque de manejo integral de cuencas hidrográficas para abordar algunos problemas de la cuenca transfronteriza Grijalva (Musálem-Castillejos, Cámara-Córdova, Laíno-Guanes, González-Espinosa y Ramírez-Marcial, 2014). Se partió del supuesto de que los problemas de la cuenca debían ser atendidos de una manera integral, al considerar de manera más amplia e interrelacionada el territorio de la cuenca con la identificación de posibles causas de los problemas en las partes bajas, tales como el cambio de curso de los ríos y las inundaciones prolongadas, al considerar fenómenos que ocurren en las partes altas, como deforestación, siembra de cultivos anuales y mantenimiento de potreros en laderas empinadas, deslizamiento de laderas, entre otras. Este enfoque integral difiere de soluciones basadas en costosas obras de ingeniería civil como las grandes represas hidroeléctricas y la canalización de los cauces que han hecho de la cuenca del río Grijalva la más intervenida en México (Arellano-Monterrosas, 2014).

El proyecto tuvo un importante apoyo del Fondo Institucional de Fomento Regional para el Desarrollo Científico, Tecnológico y de Innovación (Fordecyt) del Consejo Nacional de Ciencia y Tecnología (Conacyt) (González-Espinosa y BrunelManse, 2014). Los temas centrales incluyeron la restauración hidrológica y forestal, además de agricultura, solares y huertos familiares, ganadería orgánica, economía, toxicología ambiental, manejo de suelos, manejo de manglares, historia de las inundaciones, así como perspectiva de género y vulnerabilidad y riesgo como temas transversales. A lo largo de la cuenca se identificaron 3 «módulos regionales» en los que se concentraron los esfuerzos de más de 25 investigadores de 6 instituciones regionales y nacionales (en total participaron más de 150 personas entre personal académico y estudiantes de licenciatura y posgrado; González-Espinosa y Brunel-Manse, 2014). Debe destacarse la empatía y coincidencia en objetivos, y posterior sinergia poco usual, que se establecieron desde el período de gestación del proyecto entre las instituciones académicas y una de las secretarías del gobierno del estado de Tabasco (la entonces Secretaría de Recursos Naturales y Protección Ambiental, Sernapam).

El proyecto identificó el módulo I en la región alta de la cuenca, a la que se hace referencia en este trabajo, pero también se efectuaron actividades similares en el módulo II en los municipios contiguos de Huitiupán (Chiapas) y Tacotalpa (Tabasco); el módulo III del proyecto incluyó municipios costeros de Tabasco circundantes de la laguna de Mecoacán. En cada módulo se trabajó con al menos 8 comunidades para tener representación de las principales condiciones ambientales y socioeconómicas (González-Espinosa y Brunel-Manse, 2014; Plascencia-Vargas, González-Espinosa, Ramírez-Marcial, Álvarez-Solís y Musálem-Castillejos, 2014); no obstante, por razones logísticas y de capacidad del grupo de trabajo, se excluyeron otras áreas muy importantes como la región central de Chiapas, donde se encuentran las represas hidroeléctricas de La Angostura, Chicoasén, Malpaso y Peñitas. 
La restauración forestal e hidrológica fue abordada en los módulos I y II. Las motivaciones esenciales tuvieron que ver con la percepción a priori de la necesidad de promover la recuperación de los bosques para atenuar los riesgos de deslizamientos de laderas y cambios de cauces de los ríos, aunque también se abordó el tema de la restauración riparia en áreas poco inclinadas (Ramírez-Marcial, González-Espinosa, MusálemCastillejos, Noguera-Savelli y Gómez-Pineda, 2014). Como se describe abajo, esta prioridad, establecida desde los objetivos más amplios del proyecto, tuvo que enfrentarse a otras circunstancias que impusieron la necesidad de ajustes.

\section{Definición e implementación de las acciones}

Después de 3 meses de trabajo antropológico preliminar en cada módulo regional se incorporó una persona («técnico residente») con presencia permanente en la región, quien a través de la confianza construida con las familias, líderes y autoridades diversas logró gestionar el acceso a las comunidades dispuestas a participar. Una tarea inicial se enfocó a conocer las condiciones $\mathrm{y}$ aspiraciones prevalecientes en las comunidades con base en la metodología de medios o modos de vida («rural livelihoods»; DFID, 1999; Gómez-Pineda et al., 2014; Parra-Vázquez et al., 2009). Se realizaron talleres con miembros de familias «típicas» en cada comunidad para identificar sus necesidades, motivaciones y aspiraciones a través de la valoración de lo que constituyen 5 capitales comunitarios: natural, financiero, social, humano, y físico o de infraestructura (Gómez-Pineda et al., 2014).

Quienes participaron en los talleres reconocieron como una de sus primeras necesidades el recuperar la cobertura forestal para asegurar agua en los manantiales y la protección de las laderas para evitar su deslizamiento bajo lluvias intensas. Sin embargo, surgió claramente desde el inicio la necesidad de considerar el suministro diario de leña como una condición indispensable y prioritaria para llegar más adelante, en segunda o tercera prioridad, a los objetivos de la restauración forestal ligados a la conservación de los suelos, el agua y la biodiversidad. Las comunidades decidieron establecer una plantación de restauración con las especies que identificaron como de su interés, con base en la calidad de su leña.

Una vez definidas las especies de interés, se ejecutaron talleres para la construcción de las capacidades necesarias en temas como la cosecha y el manejo de semillas y el establecimiento de viveros, materias en las cuales el grupo de ECOSUR ha desarrollado una experiencia considerable (Ramírez-Marcial, Camacho-Cruz y González-Espinosa, 2005; Ramírez-Marcial, Camacho-Cruz, González-Espinosa y López-Barrera, 2006; Ramírez-Marcial et al., 2012, 2014). Junto con la identificación de las áreas potenciales para la restauración se realizó un estudio sobre la heterogeneidad de los suelos y así intentarla con especies y prácticas adecuadas a las variantes edáficas y de calidad de sitio (Gaspar-Santos, González-Espinosa, Ramírez-Marcial y Álvarez-Solís, 2015; Gómez-Pineda, 2012; Gómez-Pineda et al., 2014). Buscamos con esto evitar el frecuente error de los planes oficiales de restauración y de las plantaciones de reforestación, que utilizan de manera indiscriminada en todos los tipos de sitios especies que no son nativas de la región ni representan interés para las comunidades (Ramírez-Marcial et al., 2012, 2014).

Para las plantaciones de restauración la comunidad eligió lotes ligados a las escuelas y los llamados «astilleros», los cuales son predios con alguna cobertura forestal, generalmente muy degradada, de donde se extrae la leña mediante acuerdos. Aunque las comunidades identificaron las áreas desmontadas dedicadas a la agricultura de milpa o para potreros como aquellas con mayores riesgos, en ningún caso decidieron establecer ahí una plantación de restauración. Al final del proyecto asociamos estas áreas con el mayor riesgo de erosión y de posibles deslizamientos provocados por sismos y lluvias extremas (Gómez-Pineda, 2012; Gómez-Pineda et al., 2014), como las de los huracanes de 1998 (Mitch) y 2005 (Stan), y otros más frecuentes, de menor intensidad regional pero también con grandes efectos locales.

Al cabo de los 2 años del proyecto fue claro que, sin importar el grado de capacidad técnica que es posible poner a disposición de una iniciativa de intervención en comunidades empobrecidas, a su vez sustentado en investigación ecológica pertinente al objetivo, el esfuerzo puede encontrar fuertes e imprevistos obstáculos. Fue infranqueable el encuentro con la necesidad de la población local de adoptar los programas gubernamentales de transferencias entonces vigentes, que apoyaron la siembra de cultivos y la ganadería en potreros en laderas inclinadas, antagónicos con lo pretendido en la restauración hidrológica y forestal (Gómez-Pineda et al., 2014).

\section{Investigación participativa en otros temas y la implementación de un diplomado}

En su primer año el proyecto efectuó numerosas y diversas acciones de acercamiento y construcción de capacidades en las comunidades. Después de un año se concretó un riesgo inherente al trabajo de grandes grupos multidisciplinarios, al darse las tareas de los subgrupos de manera poco coordinada y a menudo sobrepuesta sobre las mismas familias; como consecuencia, las personas expuestas a contenidos particulares en los diferentes talleres no alcanzaban a ver con claridad cuál podría ser el objetivo general del proyecto.

Como resultado de esta percepción y reflexión, durante el segundo año se acordó integrar las acciones de construcción de capacidades mediante un diplomado impartido a líderes de las comunidades (Brunel-Manse, Vázquez-Nava, Mariaca-Méndez y Musálem-Castillejos, 2014) y con la participación voluntaria del personal del proyecto. El diplomado «Hacia la construcción de capacidades para la gestión integral de nuestra cuenca» se impartió durante 12 semanas en los módulos I y II, con la participación de 25-30 personas en cada caso. En cuanto a restauración en el diplomado, se partió desde construir un concepto propio del proceso con base en el aprecio de la diversidad arbórea local, el fenómeno de sucesión ecológica secundaria dirigida, junto con el adiestramiento para la recolección de semillas y de especímenes para herbarios (Ramírez-Marcial et al., 2005, 2006, 2012, 2014). A esto siguieron temas sobre germinación, tratamientos 
a las semillas para romper su posible latencia, establecimiento de almácigos y viveros y el cuidado de las plántulas y juveniles.

La comunidad Libertad Frontera, municipio de Mazapa de Madero, decidió en el inicio no participar en el proyecto. Sin embargo, como efecto lateral del diplomado fueron convencidos por la experiencia de quienes participaron para establecer por cuenta propia un vivero comunitario con especies arbóreas nativas. Los efectos del diplomado han dependido también de la posibilidad de dar continuidad a las acciones iniciadas con las comunidades. Así, con base en apoyo de la Dirección General de Corredores Biológicos de la Comisión Nacional para el Conocimiento y Uso de la Biodiversidad (Conabio), en el módulo II se ha seguido un notable caso de empoderamiento sobre las decisiones de desarrollo y gestión del ambiente por el grupo «Colectivo Almandros por un mundo mejor», con el que se mantiene colaboración intermitente pero firme en cada ocasión.

\section{Síntesis y reflexiones}

Estas 2 experiencias de IPEFA desarrolladas en comunidades empobrecidas de 2 regiones de la Sierra Madre de Chiapas permiten intentar la identificación de algunas conclusiones y «lecciones aprendidas». Salta a la vista que los procesos sobre los cuales se diseñó la participación de los grupos de académicos se imbricaron con los intereses de diversos actores. ¿Cómo somos convocados a participar los académicos practicantes de la ecología en diferentes territorios campesinos a través de la IPEFA? ¿Cómo asumir plenamente que la resolución de preguntas sobre lo que se podría llamar «práctica de la investigación ecológica» es solo una de las disciplinas que deben participar en proyectos de restauración en comunidades rurales empobrecidas?

La restauración de los bosques y predios silvopastoriles y agroforestales es apreciada desde los primeros intercambios con las comunidades como una de sus más sentidas necesidades, pero en cuanto se asocia la provisión de servicios y bienes esenciales para ellas como cosechar agua, mantener los manantiales, retener suelo, o el aprovisionamiento de leña, la restauración pasa, en la práctica y conforme se avanza hacia el establecimiento de compromisos mutuos entre ellas y los grupos académicos, a segundo o tercer plano de prioridad. Esto se ha evidenciado cuando se trata de decidir sobre la asignación de áreas para la restauración forestal ante la necesidad de mantener los terrenos para los cultivos de temporal y de supeditar el crecimiento de los árboles a lo que pueda quedar de ellos después de cubrir las enormes necesidades de leña cercana a las viviendas. Lo mismo ocurre cuando se decide privilegiar el pastoreo del ganado y los rendimientos del café por encima de la conservación y restauración de árboles silvestres en potreros y cafetales.

En un proceso de mediano o largo aliento, como lo implica necesariamente la IPEFA, es claro que nosotros mismos como académicos y funcionarios en nuestras instituciones debiéramos pugnar por la definición de convocatorias, que con criterios e indicadores muy estrictos del desempeño gradual de los proyectos, pudieran permitir el planteamiento de metas a plazos mayores de 2 o 3 años. Sin duda, la comunidad mexicana que practica la ecología forestal y agroforestal ha dado, con y como otros colegas en el resto del mundo, pasos muy importantes en su esfuerzo por ampliar sus objetivos, por identificar el contexto y los determinantes sociales de los procesos ecológicos que investiga, por propiciar o aceptar la multi y transdisciplina, y por construir con otros actores los conceptos, métodos y acciones útiles para afrontar y transformar las crisis socioambientales contemporáneas en los territorios campesinos (Castillo y Toledo, 2000; Martínez et al., 2006). Eduardo Galeano (1986) nos recuerda que en 2018 se cumplirá un siglo del surgimiento en Córdoba, Argentina, de la llamada 'universidad latinoamericana', un modelo de institución educativa que asume un compromiso para transformar desde lo profundo a la sociedad que la sustenta y la apoya para arribar a un mejor bienestar general. Quienes formamos la comunidad ecológica mexicana hemos avanzado en ubicarnos como actores sociales, pero persisten fuertes impedimentos desde la propia academia y externos que todavía debemos compartir y resolver para dar plenitud a esta visión de futuro.

\section{Agradecimientos}

Agradecemos a numerosos colegas, a quienes no es posible mencionar de manera individual, que nos han compartido a través de sus respectivas trayectorias profesionales como ecólogos sus experiencias y perspectivas acerca de la IPEFA. En especial, MGE agradece a Neptalí Ramírez Marcial por compartir la construcción de un concepto de restauración de bosques y junto con Ramón Mariaca Méndez y Claudia Brunel-Manse, la experiencia del diplomado, así como a Samuel I. Levy Tacher y Manuel R. Parra-Vázquez por compartir su visión sobre la restauración y el desarrollo en comunidades mayas de Chiapas. MGE agradece a la Secretaría de Recursos Naturales y Protección Ambiental (Sernapam) del estado de Tabasco por aportar fondos concurrentes para el proyecto Fordecyt 143303 «Gestión y estrategias de manejo sustentable para el desarrollo regional en la cuenca transfronteriza Grijalva» (2010-2013), y a la Dirección General de Corredores Biológicos de la Conabio por su apoyo para dar continuidad al trabajo en la región de La Sierra (Tabasco) durante 2013 y 2014. Las experiencias en la parte alta de la cuenca del Grijalva no habrían sido posibles ni tan fructíferas (pese a la deuda que aún prevalece por los académicos) sin la entusiasta participación y afectuosa hospitalidad de que fuimos objeto en las comunidades de los municipios de Motozintla de Mendoza y Mazapa de Madero, Chiapas, y tampoco si no hubiéramos contado con el «espejo» de experiencias precedentes vividas en comunidades de los municipios de Tacotalpa (Tabasco) y Huitiupán (Chiapas). LGB agradece a Juana Cruz-Morales por reorientar continua, crítica y constructivamente -con gran sensibilidad, respeto y solidaridad hacia la población local- los esfuerzos realizados por estudiantes e investigadores para desarrollar la IPEFA en la CART; a las decenas de mujeres y hombres habitantes de la CART que se han involucrado a lo largo de una década en el proceso; a las 21 personas que han realizado tesis de licenciatura, maestría y doctorado y estancias posdoctorales en colaboración con el proyecto (ver literatura citada), así como a sus comités de asesores de ECOSUR, UNAM, 
UASLP, Universidad de Puerto Rico, Wageningen University, Cambridge University y Columbia University; a los colegas de las redes sierra de Villaflores, Aridnet, Agro-Diversitas, Commod, IASC, COMMOD y Open-ABM. Al Conacyt por aportar fondos a través de 5 proyectos consecutivos (Ciencia Básica, UC-MEXUS, FOMIX, FORDECYT (REDISA), y SEP-SEB) y a ECOSUR por aportar fondos a través de su Departamento de Agricultura, Sociedad y Ambiente y del proyecto multidisciplinario y transversal de Agricultura familiar (20152018).

\section{Referencias}

Arellano-Monterrosas, J. L. L. (2014). Políticas públicas para la gestión de la cuenca del río Grijalva. En M. González-Espinosa y M. C. Brunel-Manse (Eds.), Montañas, pueblos y agua. Dimensiones y realidades de la cuenca Grijalva (pp. 213-239). México D.F.: Juan Pablos Editores/ECOSUR.

Astier, M., García-Barrios, L., Masera-Cerutti, O., Galván-Miyoshi, Y. y González-Esquivel, C. (2012). Assessing the sustainability of small-farmer natural resource management systems. A critical analysis of the MESMIS program (1995-2010). Ecology and Society, 17, 25.

Ayarza, M., Huber-Sannwald, E., Herrick, J. E., Reynolds, J. F., García-Barrios, L., Welchez, L. A., et al. (2010). Changing human-ecological relationships and drivers using the Quesungual agroforestry system in western Honduras. Renewable Agriculture and Food System, 25, 219-227.

Braasch, M., García-Barrios, L., Ramírez-Marcial, N., Huber-Sannwald, E. y Cortina-Villar, S. (2017). Can cattle grazing substitute fire for maintaining appreciated pine savannas at the frontier of a montane forest biospherereserve? Agriculture, Ecosystems and Environment, 250, 59-71.

Breedlove, D. E. (1981). Flora of Chiapas. Part I. Introduction to the flora of Chiapas. San Francisco, Ca: California Academy of Sciences.

Brunel-Manse, M. C. y García-Barrios, L. (2011). Acknowledging consensus and dissent among and within stakeholder groups over conservation, production and urbanization in a Mexican "Man \& the Biosphere" Reserve. Research Journal of Biological Sciences, 6, 457-469.

Brunel-Manse, M. C., Vázquez-Nava, L., Mariaca-Méndez, R. y MusálemCastillejos, K. (2014). Una experiencia de fortalecimiento de capacidades locales. El diplomado "Hacia la construcción de capacidades para la gestión integral de nuestra cuenca". En M. González-Espinosa y M. C. Brunel-Manse (Eds.), Montañas, pueblos y agua. Dimensiones y realidades de la cuenca Grijalva (pp. 677-694). México D.F.: Juan Pablos Editores/ECOSUR.

Buhmann, K. y García-Barrios, L. (2014). Aboveground dry matter production and use of fodder tree leaves (Glyricidia sepium), broad-leave herbs and invasive grasses in an experimental on-farm plantation at La Sepultura Bioreserve, Chiapas, Mexico. (Tesina). Leeuwarden, Países Bajos: ECOSUR-Hogeschool VHL, University of Applied Sciences.

Caballero, L., Macías, J., García-Palomo, A., Saucedo, G. R., Borselli, L., Sarocchi, D., et al. (2006). The September 8-9, 1998 rain-triggered flood events at Motozintla, Chiapas, Mexico. Natural Hazards, 39, 103-126.

Castillo, A. y González-Gaudiano, E. (2009). La educación ambiental para el manejo de ecosistemas: el papel de la investigación científica en la construcción de una nueva vertiente educativa. En A. Castillo y E. GonzálezGaudiano (Eds.), Educación ambiental y manejo de ecosistemas en México (pp. 9-34). México D.F.: Semarnat- INE/UNAM.

Castillo, A. y Toledo, V. (2000). Applying ecology in the Third World: the case of Mexico. BioScience, 50, 66-76.

Chappell, M. J., Wittman, H., Bacon, C. M., Ferguson, B. G., García-Barrios, L., García-Barrios, R., et al. (2013). Food sovereignty: an alternative paradigm for poverty reduction and biodiversity conservation in Latin America. F1000 Research, 2, 235.

Conagua (Comisión Nacional del Agua). (2013). Datos climáticos históricos de las estaciones meteorológicas de Motozintla de Mendoza y Buenos Aires. Tuxtla Gutiérrez, Chiapas: Comisión Nacional del Agua, Secretaría de Medio Ambiente y Recursos Naturales.
Cruz-Morales, J. (2014a). Construcción de territorios ambientales mediante procesos de aprendizaje social. El caso de la cuenca alta del río El Tablón, Reserva de la Biosfera La Sepultura, Chiapas, México (Tesis doctoral). México D.F.: Universidad Autónoma Metropolitana.

Cruz-Morales, J. (2014b). Desafíos para construir la democracia ambiental en la Cuenca Alta del río El Tablón (CART), Reserva de la Biosfera La Sepultura (REBISE), Chiapas, México. En M. Legorreta-Díaz, M. Márquez-Rosano, y T. Trench (Eds.), Paradojas de las tierras protegidas en Chiapas. Democracia y política ambiental en Reservas de Biosfera en Chiapas (pp. 21-60). México D.F.: CEIICH-CRIM-UNAM; DCRU-UACh.

Cruz-Morales, J., Trujillo-Vázquez, R., García-Barrios, L., Ruiz-Rodríguez, J. M. y Jiménez-Trujillo, J. A. (2011). Buenas prácticas para la ganadería sustentable en la Reserva de la Biosfera La Sepultura. (REBISE). Texcoco, Edo. de México: Universidad Autónoma Chapingo, El Colegio de la Frontera Sur, Conservación Internacional-México y Comisión de Áreas Naturales Protegidas.

DFID (Department for International Development). (1999). Sustainable livelihoods framework guide. Londres: Department for International. Recuperado 30 Sept 2010 de http://www.eldis.org/go/topics/ dossiers/livelihoods-connect/what-are-livelihoods-approaches/training-andlearning-materials

Etienne, M. (2014). Companion modelling: a participatory approach to support sustainable development. Berlín: Springer.

Fortmann, L. (2008). Participatory research in conservation and rural livelihoods: doing science together. Hoboken, N.J.: Wiley \& Blackwell.

Galeano, E. (1986). Memoria del fuego 3. El siglo del viento. México D.F.: Siglo XXI.

García-Barrios, L. (2010). LASARUS. (Let Agroforests Sustain Agrarian Regions Under Stress) Interactive, user friendly Agent Based Model. NETLOGO 4.1.1 language. ECOSURDisco Compacto. Codigo disponible mediante solicitud al autor (lgarcia@ecosur.mx).

García-Barrios, L., Álvarez-Solís, D., Brunel-Manse, M. C., Cruz-Morales, J., García-Barrios, R., Hernández-Ramírez, F., et al. (2012). Innovación socioambiental en la cuenca alta del río El Tablón (CART), sierra de Villaflores, Chiapas. Objetivo, estrategia y métodos de investigación-acción participativa. En E. Bello-Baltazar, E. Naranjo-Piñera, y E. J. Vandame (Eds.), La otra innovación para el ambiente y la sociedad en la Frontera Sur de México (pp. 145-170). San Cristóbal de Las Casas, Chiapas: El Colegio de la Frontera Sur.

García-Barrios, L., Cruz-Morales, J., Vandermeer, J. y Perfecto, I. (2017). The Azteca chess experience: learning how to share concepts of ecological complexity with small coffee farmers. Ecology and Society, 22, 37.

García-Barrios, L., Galván-Miyoshi, Y., Valdivieso-Pérez, A., Masera, O., Bocco, G. y Vandermeer, J. (2009). Neotropical forest conservation, agricultural intensification and rural out-migration: the Mexican experience. Bioscience, 59, 863-873.

García-Barrios, L., García-Barrios, R., Cruz-Morales, J. y Smith, J. A. (2015) When death approaches: reverting or exploiting emergent inequity in a complex land-use table-board game. Ecology and Society, 20, 13.

García-Barrios, L., García-Barrios, R., Waterman, A. y Cruz-Morales, J. (2011). Social dilemmas and individual/group coordination strategies in a complex rural land-use game. International Journal of the Commons, 5, 364-387.

García-Barrios, L., Perfecto, I. y Vandermeer, A. (2016). Azteca chess: gamifying a complex ecological process of autonomous pest control in shade coffee. Agriculture. Ecosystems and Environment, 232, 190-198.

García-Barrios, L., Trujillo-Vásquez, R., Cruz-Morales, J., Waterman, A. G. y García-Barrios, R. (2010). Diseño y establecimiento participativo de sistemas de producción de árboles forrajeros en los potreros bajo pastoreo intensivo de la cuenca alta del río El Tablón (CART), REBISE, Chiapas. En E. Bello-Baltazar, E. Naranjo-Piñera, y E. J. Vandame (Eds.), Innovación socioambiental y desarrollo en la frontera sur de México (pp. 30-33). San Cristóbal de Las Casas: El Colegio de la Frontera Sur. Red de Espacios de Innovación Socioambiental.

Gaspar-Santos, E. S., González-Espinosa, M., Ramírez-Marcial, N. y ÁlvarezSolís, J. D. (2015). Acumulación y descomposición de hojarasca en bosques secundarios del sur de la sierra Madre de Chiapas, México. Bosque, 36, $467-480$. 
Gershenson, C., Aerts, D. y Edmonds, B. (2007). Philosophy and complexity. Worldviews science and us. Singapur: World Scientific.

Gómez-Pineda, E. (2012). Estrategias para la restauración forestal en comunidades del municipio de Motozintla, Chiapas, México (Tesis de Maestría en Ciencias). San Cristóbal de Las Casas, Chiapas, México: El Colegio de la Frontera Sur.

Gómez-Pineda, E., González-Espinosa, M., Parra-Vázquez, M. R., DíazHernández, B. M., Musálem-Castillejos, K. y Ramírez-Marcial, N. (2014). Medios de vida y condicionantes que enfrenta la restauración forestal: experiencias en la cuenca alta del río Grijalva, Chiapas. En M. González-Espinosa у M. C. Brunel-Manse (Eds.), Montañas, pueblos y agua. Dimensiones y realidades de la cuenca Grijalva (pp. 257-282). México D.F.: Juan Pablos Editores/ECOSUR.

González-Espinosa, M. y Brunel-Manse, M. C. (2014). Introducción. En M. González-Espinosa y M. C. Brunel-Manse (Eds.), Montañas, pueblos y agua. Dimensiones y realidades de la cuenca Grijalva. (pp. 15-26). México D.F. Juan Pablos Editores/ECOSUR.

González-Espinosa, M. y Ramírez-Marcial, N. (2013). Comunidades vegetales terrestres. En Comisión Nacional para el Conocimiento y Uso de la Biodiversidad y Gobierno del Estado de Chiapas (Eds.), La biodiversidad en Chiapas: estudio de estado, Vol. 2. (pp. 21-42). México D.F.: Conabio.

Gutiérrez-Navarro, A. (2015). Perspectivas campesinas sobre el uso y manejo del fuego en la Reserva de la Biosfera de la Sepultura. Chiapas (Tesis de Maestría en Ciencias). San Cristóbal de Las Casas, Chiapas, México: El Colegio de la Frontera Sur.

Heras, M., Tabara, D. y Meza, A. (2016). Performing biospheric futures with younger generations: a case in the MAB Reserve of La Sepultura, Mexico. Ecology and Society, 21, 14

Hernández-Moreno, M. G. (2011). Procesos de remoción en masa en el municipio de Motozintla de Mendoza, Chiapas (Tesis). México D.F.: Facultad de Filosofía y Letras, Universidad Nacional Autónoma de México.

Inegi (Instituto Nacional de Estadística y Geografía). (1985). Cartas temáticas 1:250 000. Información digital en formato shp. San Cristóbal de Las Casas, Chiapas, México: LAIGE-ECOSUR.

Inegi (Instituto Nacional de Estadística y Geografía). (1988). Carta Geológica y Carta Edafológica 1:250 000. Información digital en formato shp. San Cristóbal de Las Casas, Chiapas, México: LAIGE-ECOSUR.

Inegi (Instituto Nacional de Estadística y Geografía). (2013). Simulador de flujos de agua de cuencas hidrológicas (SIATL) [consultado 14 Mar 2014]. Disponible en: http://antares.inegi.org.mx/analisis/red_hidro/SIATL/?s=geo\&c=1693\#

Jackson, L. E., Pulleman, M. M., Brussard, L., Bawa, K. S., Brown, G. G., Cardoso, I. M., et al. (2012). Social-ecological and regional adaptation of agrobiodiversity management across a global set of research regions. Global Environmental Change, 22, 623-639.

Laíno-Guanes, R. M., Bello-Mendoza, R., González-Espinosa, M., RamírezMarcial, N., Jiménez-Otárola, F. y Musálem-Castillejos, K. (2015). Concentración de metales en agua y sedimentos de la cuenca alta del río Grijalva, frontera México-Guatemala. Tecnología y Ciencias del Agua, 6, $61-74$

Laíno-Guanes, R. M., González-Espinosa, M., Ramírez-Marcial, N., BelloMendoza, R., Jiménez, F., Casanoves, F., et al. (2016). Human pressure on water quality and water yield in the upper Grijalva river basin in the Mexico-Guatemala border. Ecohydrology \& Hydrobiology, 95, 1-11.

Laíno-Guanes, R. M., Musálem-Castillejos, K., González-Espinosa, M. y Ramírez-Marcial, N. (2014). El uso del agua en Motozintla de Mendoza, Chiapas: conflictos. contaminación y posibles soluciones. En M. González-Espinosa y M. C. Brunel-Manse (Eds.), Montañas, pueblos y agua. Dimensiones y realidades de la cuenca Grijalva. (pp. 445-462). México D.F.: Juan Pablos Editores/ECOSUR.

Larson, A. y Ribot, J. (2007). The poverty of forestry policy: double standards on an uneven playing field. Sustainability Science, 2, 189-204.

Martínez, M. L., Manson, R. H., Balvanera, P., Dirzo, R., Soberón, J., GarcíaBarrios, L., et al. (2006). The evolution of ecology in Mexico: facing challenges and preparing for the future. Frontiers in Ecology and the Environment, 5, 259-267.

Meza-Jiménez, A. (2012). Nuevas herramientas de educación ambiental infantil para conservar la vegetación riparia y su biodiversidad en la zona de amor- tiguamiento de la REBISE, Chiapas (Tesis). Puebla, México: Benemérita Universidad Autónoma de Puebla.

Meza-Jiménez, A. y García-Barrios, L. (2015). Los juegos del PAN. Nuevas prácticas docentes para identificar las actitudes, motivaciones y decisiones de la juventud rural a través de juegos del PAN (Patrimonio agrícola/natural). Manual del Docente de la Sierra de Villaflores. Tuxtla Gutiérrez, Chiapas: ECOSUR.

Meza-Jiménez, A., García-Barrios, L., Saldívar-Moreno, A. y Vera-Noriega, J. A. (2016). Design and evaluation of educational socio-environmental games to identify attitudes, motivations and decisions of smallholder contemporary rural youth. EDUCARE, 20, 1-36.

Morales-Díaz, C. (2011). Evaluación experimental de prácticas para establecer plantaciones de Glyricidia sepium en la REBISE, Chiapas (Tesis de maestría). El Colegio de la Frontera Sur: San Cristóbal de Las Casas Chiapas, México.

Musálem-Castillejos, K., Cámara-Córdova, J., Laino-Guanes, R., GonzálezEspinosa, M. y Ramírez-Marcial, N. (2014). Manejo integral de cuencas hidrográficas. (MICH): el enfoque utilizado en el proyecto FORDECyT Cuenca Grijalva. En M. González-Espinosa y M. C. Brunel-Manse (Eds.), Montañas, pueblos y agua. Dimensiones y realidades de la cuenca Grijalva (pp. 80-102). México D.F.: Juan Pablos Editores/ECOSUR.

Newig, N. y Fritsch, O. (2009). Environmental governance: participatory, multi-level - and effective? Environmental Policy and Governance, 19, 197-214.

Oleta-Barrios, J. (2012). Adopción del vermicomposteo para establecer árboles forrajeros en la zona de amortiguamiento de la REBISE, Chiapas. México (Tesis de Maestría en Ciencias). San Cristóbal de Las Casas, Chiapas, México: El Colegio de la Frontera Sur.

Ostrom, E. (2009). A general framework for analyzing sustainability of socialecological systems. Science, 325, 422-429.

Parra-Vázquez, M. R., Herrera-Hernández, O. B., Huerta-Silva, M., Ramos, P., Román, R. S. I., Liscovsky, I. J., et al. (2009). Manual de planeación comunitaria, con el enfoque de medios de vida sustentables. para promotores y facilitadores del desarrollo comunitario. San Cristóbal de Las Casas, Chiapas: El Colegio de la Frontera Sur.

Perfecto, I. y Vandermeer, J. (2015). Coffee agroecology: a new approach to understanding agricultural biodiversity, ecosystem services and sustainable development. Londres: EarthScan from Routledge.

Plascencia-Vargas, H., González-Espinosa, M., Ramírez-Marcial, N., ÁlvarezSolís, D. y Musálem-Castillejos, K. (2014). Características físico-bióticas de la cuenca del río Grijalva. En M. González-Espinosa y M. C. Brunel-Manse (Eds.), Montañas, pueblos y agua. Dimensiones y realidades de la cuenca Grijalva (pp. 29-79). México D.F.: Juan Pablos Editores/ECOSUR.

Ramírez-Marcial, N., Camacho-Cruz, A. y González-Espinosa, M. (2005). Potencial florístico para la restauración de bosques en Los Altos y Montañas del norte de Chiapas. En M. González-Espinosa, N. Ramírez-Marcial, y L. Ruiz-Montoya (Eds.), Diversidad biológica en Chiapas. (pp. 325-369). México D.F.: Plaza y Valdéz.

Ramírez-Marcial, N., Camacho-Cruz, A., González-Espinosa, M. y LópezBarrera, F. (2006). Establishment, survival and growth of tree seedlings under successional montane oak forests in Chiapas, Mexico. En M. Kappelle (Ed.), Ecology and conservation of Neotropical oak forests. (pp. 177-189). Ecological Studies Núm. 185. Berlín: Springer-Verlag.

Ramírez-Marcial, N., González-Espinosa, M., Musálem-Castillejos, K., Noguera-Savelli, E. y Gómez-Pineda, E. (2014). Estrategias para una construcción social de la restauración forestal en comunidades de la cuenca media y alta del río Grijalva. En M. González-Espinosa y M. C. Brunel-Manse (Eds.), Montañas, pueblos y agua. Dimensiones y realidades de la cuenca Grijalva (pp. 528-564). México D.F.: Juan Pablos Editores/ECOSUR.

Ramírez-Marcial, N., Luna-Gómez, A., Castañeda-Ocaña, H. E., Martínez-Icó, M., Holz, S. C., Camacho-Cruz, A., et al. (2012). Guía de propagación de árboles nativos para la recuperación de bosques. San Cristóbal de Las Casas, Chiapas: El Colegio de la Frontera Sur.

Richter, M. (2000). The ecological crisis in Chiapas: a case study from Central America. Mountain Research and Development, 20, 332-339.

Roblero-Morales, M. y Ramírez-Moreno, L. (2014). Resumen diagnóstico del Módulo regional I: microcuenca de Motozintla de Mendoza-Mazapa de Madero. En M. González-Espinosa y M. C. Brunel-Manse (Eds.), 
Montañas, pueblos y agua. Dimensiones y realidades de la cuenca Grijalva. (pp. 105-118). México D.F.: Juan Pablos Editores/ECOSUR.

Román-Cuesta, R. M., Gracia, M. y Retana, J. (2003). Environmental and human factors influencing fire trends in ENSO and non-ENSO years in tropical Mexico. Ecological Applications, 13, 1177-1192.

Rosabal-Ayan, L. (2015). Estrategias campesinas de alimentación del ganado durante el estiaje, en la CART-REBISE, Chiapas, México (Tesis de Maestría en Ciencias). San Cristóbal de Las Casas, Chiapas: El Colegio de la Frontera Sur.

Salazar, M. (1992). La investigación-acción participativa. Madrid: Editorial Popular.

Sanfiorenzo-Barnhard, C. (2012). Tree composition and vegetation structure in functional rangelands in a buffer zone of La Sepultura Bio-Reserve Chiapas, Mexico (Tesis de Maestría en Ciencias). Río Piedras, Puerto Rico: Universidad de Puerto Rico.

Sanfiorenzo-Barnhard, C., García-Barrios, L., Meléndez-Ackerman, E. y Trujillo-Vásquez, R. (2009). Woody cover and local farmers' perceptions of active pasturelands in La Sepultura Biosphere Reserve. Mountain Research and Development, 29, 320-327.

Sayer, J., Sunderland, T., Ghazoul, J., Pfund, J., Sheil, D., Meijaard, E., et al. (2013). Ten principles for a landscape approach to reconciling agriculture, conservation, and other competing land uses. Proceedings of the National Academy of Sciences of the United States of America, 110, 8349-8356.

Speelman, E., García-Barrios, L., Groot, J. y Tittonell, P. (2013). Gaming for smallholders' participation in the design of more sustainable agricultural landscapes. Agricultural Systems, 126, 62-75.

Speelman, E., Groot, J., García-Barrios, L., Kok, K., van Keulen, H. y Tittonell, P. (2014). From coping to adaptation to economic and institutional change: trajectories of change in land use management and social organization in a Biosphere Reserve community, Mexico. Land Use Policy, 41, 31-44.

Tadelis, S. (2013). Game theory: an introduction. Princeton, New Jersey: Princeton University Press.

Tenza-Peral, A., García-Barrios, L. y Giménez-Casalduero, A. (2011). Agricultura y conservación en Latinoamérica en el siglo XXI: ¿Festejamos la "transición forestal" o construimos activamente "la matriz de la naturaleza"? Interciencia, 36, 500-507.

Valdivieso-Pérez, A., García-Barrios, L., Álvarez-Solís, D. y Nahed-Toral, J. (2013). De maizales a potreros: cambio en la calidad del suelo en Los Ángeles, Villaflores, Chiapas. México. Terra Latinoamericana, 30, 1-12.

Valdivieso-Pérez, A., García-Barrios, L. y Plascencia, H. (2009). Cambio de uso del suelo en la zona de amortiguamiento de la REBISE (1975-2005): crisis del maíz. ganaderización y recuperación arbórea marginal. En B. Cavallotti, C. Marcof, y B. Ramírez (Eds.), Ganadería y seguridad alimentaria en tiempo de crisis. (pp. 349-354). Chapingo, Estado de México: Universidad Autónoma Chapingo, Campus Puebla.

Valencia, V., García-Barrios, L., West, P., Sterling, E. y Naeem, S. (2014). The role of coffee agroforestry in the conservation of tree diversity and community composition of native forests in a Biosphere Reserve. Agriculture, Ecosystems and Environment, 189, 154-163.

Valencia, V., Naeem, S., García-Barrios, L., West, P. y Sterling, E. (2016) Conservation of tree species of late succession and conservation concern in coffee agroforestry systems. Agriculture, Ecosystems and Environment, 219, 32-41.

Valencia, V., West, P., Sterling, E. J., García-Barrios, L. y Naeem, S. (2015). The use of farmers' knowledge in coffee agroforestry management: implications for the conservation of tree biodiversity. Ecosphere, 6, 1-17.

Van Hecken, G., Bastiaensen, J. y Windey, C. (2015). Towards a power-sensitive and socially-informed analysis of payments for ecosystem services (PES): addressing the gaps in the current debate. Ecological Economics, 120, $117-125$

Velázquez, A., Torres, A. y Bocco, G. (2003). Las enseñanzas de San Juan. Investigación participativa para el manejo integral de recursos naturales. México D.F.: INE-Semarnat.

Vides-Borrell, E., García-Barrios, L., Álvarez-Solís, D., Nigh, R., AstierCalderón, M. y Douterlungne, D. (2011). Establishment and growth of Gliricidia sepium in grasslands under different types of fertilization and weed management. Research Journal of Biological Sciences, 6, 468-474.

Villafuerte-Solís, D. (2010). Condiciones de vulnerabilidad productiva, económica y social. En D. Villafuerte-Solís y E. Mansilla (Eds.), Vulnerabilidad y riesgos en la sierra de Chiapas: dimensiones económica y social (pp. 79-142). Tuxtla Gutiérrez, Chiapas: Universidad de Ciencias y Artes de Chiapas.

Villafuerte-Solís, D. y García-Aguilar, M. C. (2004). Pobreza y migración en la sierra de Chiapas. Estudios Sociales y Humanísticos, 2, 81-93.

Villafuerte-Solís, D. y Mansilla, E. (2010). Vulnerabilidad y riesgos en la sierra de Chiapas: dimensiones económica y social. Tuxtla Gutiérrez, Chiapas: Universidad de Ciencias y Artes de Chiapas.

Waibel, L. (1946). La sierra Madre de Chiapas. México D.F.: Sociedad Mexicana de Geografía y Estadística.

Zabala, A. (2015). Rural livelihoods and attitudes toward silvopastoral innovations in a buffer zone of La Sepultura Bio-Reserve Chiapas, Mexico (Tesis doctoral). Cambridge, Reino Unido: Cambridge University.

Zabala, A., Pascual, U. y García-Barrios, L. (2017). Payments for pioneers? Revisiting the role of external rewards for sustainable innovation under heterogeneous motivations. Ecological Economics, 135, 234-245. 